\title{
A NEW VERSION OF THE MAIN INEQUALITY AND THE UNIQUENESS OF HARMONIC MAPS
}

\author{
By \\ V. MARKović AND M. MATELJEVIĆ
}

\section{A. Introduction and statement of main results}

A1. In this paper, we state a new version of an inequality of Reich and Strebel, namely their so-called Main Inequality, and use it to study the uniqueness property of harmonic mappings. To state the main inequality we need the following notation.

Let $D$ and $G$ be domains in $\mathbb{C}$ and let $f: D \mapsto G$ be a mapping. We use the notation

$$
d f=p d z+q d \bar{z}, \quad \text { where } p=\partial f \quad \text { and } \quad q=\bar{\partial} f .
$$

We denote the complex (Beltrami) dilatation by

$$
\mu_{f}=\operatorname{Belt}[f]=\frac{q}{p}
$$

and the dilatation by

$$
D_{f}=\frac{|p|+|q|}{|p|-|q|}
$$

It is convenient to suppose that our mappings are sense-preserving.

We say that a homeomorphism $f: D \mapsto G$ is $K$ quasiconformal if $f$ is $\mathrm{ACL}$ (absolutely continuous on lines) on $D$ and $D_{f}(z) \leq K$ a.e. on $D$.

Let $\Delta$ denote the unit disk and

$$
T_{\mu} \varphi(z)=\frac{\left|1-\mu(z) \frac{\varphi(z)}{|\varphi(z)|}\right|^{2}}{1-|\mu(z)|^{2}} .
$$

We refer to the following result as the Reich-Strebel inequality or the Main Inequality (see $[\operatorname{ReS} 1])$.

Theorem RS (Reich and Strebel). Suppose that $f$ is a quasiconformal homeomorphism of $\Delta$ onto itself which is the identity on $\partial \Delta$. Then, with $\mu=\mu_{f}$,

$$
\iint_{\Delta}|\varphi(z)| d x d y \leq \iint_{\Delta}|\varphi(z)| T_{\mu} \varphi(z) d x d y
$$


for every analytic integrable function $\varphi$ on $\Delta$.

Various forms of this result play a major role in the theory of quasiconformal mappings and have many applications. For applications to extremal and uniquely extremal quasiconformal mappings, we refer the interested reader to the book by Gardiner ([G]), and for some recent results to [MM1], [BMM], [BLMM] and [Re3].

It is convenient to explain our results first in the setting of the unit disk. Suppose that

(a) $f$ is a homeomorphism of $\bar{\Delta}$ onto itself,

(b) $f$ has first partial derivatives on $\Delta$, and

(c) $f$ is the identity on $\partial \Delta$.

Theorem 1. With the hypotheses and notation above, the inequality (A1) holds for every integrable analytic function $\varphi$ on $\Delta$.

This theorem gives a new version of the Main Inequality, which is applicable to mappings which are not quasiconformal mappings.

A2. Let $M$ and $N$ be two Riemann surfaces with local conformal metrics $\sigma|d z|^{2}$ and $\rho|d w|^{2}$ and let $f: M \mapsto N$.

It is convenient to use the notation $d f=p d z+q \overline{d z}$ in local coordinates, where $p=\partial f$ and $q=\bar{\partial} f$.

The energy integral of $f$ is

$$
E(f, \rho)=\int_{M} \rho \circ f\left(|p|^{2}+|q|^{2}\right) d x d y .
$$

A critical point of the energy functional is called a harmonic mapping. The EulerLagrange equation for the energy functional is

$$
f_{z \bar{z}}+\partial(\log \rho) \circ f p q=0 .
$$

It seems that the classical theory of elliptic equations does not suffice for a study of the Euler-Lagrange equation without the use of additional tools. In [MM2] (see also [MM3]), the current authors initiated the study of the uniqueness of solutions of equation (A2) with the help of the Main Inequality. With ordinary harmonic functions in mind, it was natural to consider the question of uniqueness of harmonic mappings, without the assumption that the mappings are of bounded dilatation, and to try to extend the range of applications of the Main Inequality. This led us to Theorem 1 and its applications. 
For basic properties of harmonic mappings and for further information on the literature, we refer to Jost [J], Schoen-Yau [SY] and Schoen [Sc].

This general notion of harmonic mappings (see Section E1 below) allows for the following:

A3. If $f$ is a harmonic mapping, then $\varphi=\rho \circ f p \bar{q} d z^{2}$ is a holomorphic quadratic differential, which is called the Hopf differential of $f$ and which is denoted by $H o p f(f)$.

For example, if $M$ and $N$ are subsets of the complex plane $\mathbb{C}$, this simply means that the function $\rho \circ f p \bar{q}$ is a holomorphic function.

A4. The following is an application of Theorem 1.

Theorem 2. (The uniqueness property of the identity). Under the hypothesis of Theorem 1, if (in addition) we suppose that $f$ is harmonic and that the Hopf differential of $f$ is nonzero and integrable on $\Delta$, then $f$ is the identity on $\bar{\Delta}$.

To state our next result, we need the notion of locally-quasiconformal mappings. Let $M$ and $N$ be two Riemann surfaces and $f: M \mapsto N$. We say that $f$ is locally quasiconformal if for every point $p \in M$ there is a neighbourhood $V$ such that the restriction of $f$ to $V$ is a quasiconformal mapping.

Theorem 3. (First uniqueness property). Suppose that $f$ and $g$ are harmonic homeomorphisms from the closed unit disk onto itself which are locally quasiconformal on $\Delta$ and suppose that $f=g$ on the boundary of the unit disk. If, in addition, the Hopf differentials of $f$ and $g$ are integrable on $\Delta$, then $f$ and $g$ are identical.

Moreover, we shall prove a uniqueness result for homotopic mappings between compact Riemann surface (see Theorem 4, the second uniqueness property, and part E4 of the section E). The idea of the proof of Theorem 3 (and Theorem 4) is to apply Theorem 1, the Main Inequality, to the functions $\varphi=\rho \circ f p \bar{q}$ and $\psi=\rho \circ g A \bar{B}$, where $A=\partial g$ and $B=\bar{\partial} g$. For further generalizations of Theorem 3 and Theorem 4, see part F5 of Section F.

Note that when we work with mappings which have first generalized derivatives (without the assumption of quasiconformality), we have new features (see below B3, and sections C, E1, E3, F4 and F5 for more details).

We start now with a short review of some previously obtained results, which are related to Theorem 3 and Theorem 4 .

A5. After we had completed an earlier version of the present paper, E. Reich pointed out to us that $\mathrm{H}$. Wei [We] studied the uniqueness property of harmonic 
mappings with the help of the Main Inequality. Also, we became aware of the paper $[\mathrm{CH}]$ of Coron and Helein.

By using the formula for the energy of variation of a mapping (see [ReS2]) and the Reich-Strebel inequality, $H$. Wei proved a weaker version of Theorem 3 concerning quasiconformal mapping under additional hypotheses that

(c) $f$ and $g$ are quasiconformal mappings from the unit disk $\Delta$ onto itself,

(d) the metric density $\rho$ is an integrable function on $\Delta$.

Note that the hypotheses (c) and (d) provide that the energy integral of $f$ and $g$ are finite.

In [CH], Coron-Helein used an approach, which is completely different from H. Wei in [We], to study minimizing harmonic mappings. Their approach was based on the decomposition of a given metric $g$ on $\Delta$ as the sum of two metrics $c$ and $h$, where $c$ is a conformal metric with respect to the euclidean metric $e, h$ has non-positive Gaussian curvature and the identity mapping, $I d$, is a harmonic mapping between $(\Delta, e)$ and $(\Delta, h)$.

Theorem CH (Coron-Helein). Let $(M, h)$ and $(N, g)$ be two Riemannian compact surfaces of class $C^{\infty}$, possibly with boundary. Then any smooth harmonic diffeomorphism between $(M, h)$ and $(N, g)$ is minimizing in its homotopy class. Moreover, if $\partial M$ is non-empty, or if the genus of $M$ is strictly larger than one, then such a diffeomorphism is the unique minimizing map in its homotopy class.

An inspection of the proofs of Theorem 2, [We], Theorem 3 and Theorem 3', $[\mathrm{CH}]$, shows that the assumption that harmonic maps are of finite energy is essential for both proofs.

Note that the assumption that $f$ and $g$ have finite energy integrals insures that Hopf differentials of $f$ and $g$ are integrable functions on $\Delta$. Thus we prove our Theorem 3 under weaker conditions. Namely, we require only that the Hopf differentials of corresponding mappings are integrable.

We refer the interested reader to $[J]$ for the global uniqueness theorem of Al'ber and Hartman and for the result of Jäger and Kaul and to the Schoen-Yau book ([SY]) for uniqueness theorems concerning harmonic maps into non-positive curved metric spaces.

A6. The structure of this paper is as follows. In Section B, we prove a version of Grötzsch's principle concerning mappings with $L^{1}$-derivatives. In Section C, we give proofs of Lemma 1 and Lemma 2, which we use in the proof of Theorem 1. In Section D, the proof of a new version of the Main Inequality (Theorem 1) is given. In Section E, we use Theorem 1 to prove the uniqueness results (Theorems 
2-4). In Section F, we give further results, comments and some applications of Theorem 2 to the case in which the energy integrals are infinite.

\section{B. The problem of Grötzsch}

B1. In order to motivate the statement and proof of our version of the Main Inequality, we shall emphasize the main points in the proof of Grötzsch's principle. We follow [MM2], where we announced the results of this section.

If $Q$ is a square and $R$ is a rectangle, not a square, there is no conformal mapping of $Q$ on $R$ which maps vertices on vertices. Instead, Grötzsch asked for the most nearly conformal mapping of this kind and took the first step toward the creation of a theory of quasiconformal mappings.

Let $w=f(z)$ be a mapping from one region to another. Recall that

$$
d f=p d z+q d \bar{z}, \quad \text { where } p=\partial f \quad \text { and } \quad q=\bar{\partial} f .
$$

The complex (Beltrami) dilatation is

$$
\mu_{f}=\operatorname{Belt}[f]=\frac{q}{p}
$$

and the dilatation of $f$ is

$$
D_{f}=\frac{|p|+|q|}{|p|-|q|} .
$$

We pass to the Grötzsch problem and give it a precise meaning by saying that $f$ is most nearly conformal if sup $D_{f}$ is as small as possible.

Let $R, R^{\prime}$ be two rectangles with sides $a, b$ and $a^{\prime}, b^{\prime}$. We may assume that $K=b^{\prime} / a^{\prime}: b / a \geq 1$. The mapping $f$ is supposed to be $C^{1}$-homeomorphism from $\bar{R}$ onto $\overline{R^{\prime}}$, which takes $a$-sides into $a$-sides and $b$-sides into $b$-sides.

Next, let $\Gamma_{x}$ be the vertical segment which is the intersection of the line $\operatorname{Re} z=x$ with $\bar{R}$ and $\gamma_{x}$ the curve which is image of $\Gamma_{x}$ under $f$. The starting point of Grötzsch's approach is the obvious geometric inequality

$$
b^{\prime} \leq \text { length }\left(\gamma_{x}\right)=\int_{0}^{b}|p-q| d y .
$$

Using

$$
\iint_{R} J_{f} d x d y=a^{\prime} b^{\prime}
$$

where $J_{f}$ denotes the Jacobian of $f$, and the Cauchy-Schwarz inequality, one gets

$$
K \leq \sup D_{f}
$$


The minimum is attained for the affine mapping.

The restriction to $C^{1}$-mapping is not essential. The inequality (B3) holds for quasiconformal mapping (see, for example, $[\mathrm{Ah}]$ ).

In order to give a version of Grötzsch's principle concerning mappings with $L^{1}$-derivatives, we need the following definition.

B2. Definition of $L^{p}$-derivatives. Let $D$ be a domain in $C$. We say that a function $f: D \mapsto C$ has $L^{p}$-derivatives, $p \geq 1$, if it satisfies the following two conditions:

(a) $f$ is absolutely continuous on lines in $D$, and

(b) the partial derivatives $f_{x}$ and $f_{y}$ belong to $L^{p}$ on every compact subset of $D$.

When we say that $f$ has generalized first derivatives in $D$, this means that $f$ has $L^{1}$-derivatives in $D$. For various characterizations of functions with $L^{p_{-}}$ derivatives and their important role in the theory of quasiconformal mappings, we refer to Chapters III to VI of the book by Lehto-Virtanen ([LV]).

B3. A version of Grötzsch's principle. Before we give a further extension of Grötzsch's principle, it is useful to consider the following example, in which (B1) and (B3) do not hold.

Example 1. Let $\alpha: I \mapsto I$, where $I=[0,1]$, be the Cantor function and let $f(z)=x+i(y+\alpha(y))$.

Note that this function does not satisfy the ACL property and that the known formula for the length of curve by means of first partial derivatives does not hold.

Suppose that

(a) $f$ is a homeomorphism of the closed rectangle $\bar{R}$ onto the closed rectangle $\overline{R^{\prime}}$ which maps $a$-sides onto $a^{\prime}$-sides and $b$-sides onto $b^{\prime}$-sides, and

(b) $f$ has generalized first derivatives on $R$.

In order to obtain our conclusion, we can follow the outline of the proof of Grötzsch's principle from part B1 of this section.

We need the following definition.

At a point $z$ where $\mu(z)$ is defined and $|\mu(z)| \neq 1$ we define $T_{\mu}(z)$ by

$$
T_{\mu}(z)=\frac{|1-\mu(z)|^{2}}{1-|\mu(z)|^{2}}
$$

Also, at point $z$ where $|p(z)|=|q(z)|$ we define $T_{\mu}(z)$ to be zero if $p(z)=q(z)$ and $+\infty$ if $p(z) \neq q(z)$. 
Now, we can give the precise meaning of $T_{\mu} \varphi$ by means of $T_{\chi}$, where

$$
\chi=\mu \varphi /|\varphi| \text {. }
$$

Since $f$ satisfies the ACL-property, inequality (B1) holds for a.a. $x \in[0, a]$.

In order to prove inequality (B4) (see below), we can suppose that $T_{\mu}$ is defined and finite a.e. on $R$, because otherwise the right-hand side of (B4) is infinite. Next, we integrate with regard to $d x$ over $[0, a]$ and use the fact that the Jacobian

$$
J_{f}=|p|^{2}\left(1-|\mu|^{2}\right) \text { a.e. on } R \text {. }
$$

Instead of (B2), we have

$$
\int_{R} J_{f} d x d y \leq \operatorname{area}\left(R^{\prime}\right)=a^{\prime} b^{\prime} .
$$

Now, an application of the Cauchy-Schwarz inequality gives

$$
\operatorname{area}(R) \frac{b^{\prime}}{b} \leq\left[\operatorname{area}\left(R^{\prime}\right)\right]^{1 / 2}\left[\iint_{R} T_{\mu}\right]^{1 / 2}
$$

Further development of the ideas outlined above leads us to Lemma 1 (see below), which will be used in the proof of the new version of the main inequality.

\section{Proofs of Lemma 1 and Lemma 2}

Let $D$ be a vertically convex domain of finite area in the complex plane $\mathbb{C}$ and let $F$ be a mapping from the domain $D$ onto the domain $G$.

Suppose that we have metric $d s=\rho(w)|d w|$ on $G$.

Let $\Gamma_{x}$ be the interval which is the intersection of $D$ with the straight line $\operatorname{Re} z=x$, and let $\gamma_{x}$ be the curve which is the image of $\Gamma_{x}$ under $F$. Let $p(x, y)=x$ be the projection and let $(\alpha, \beta)=p(D)$.

Lemma 1. With the notation and hypothesis just stated, suppose (in addition) that the mapping $F$ is homeomorphism which has generalized first derivatives and that

$$
\operatorname{length}\left(\Gamma_{x}\right) \leq \int_{\gamma_{x}} \rho(w)|d w| \quad \text { a.e. in }(\alpha, \beta)
$$


Then

$$
\operatorname{area}(D) \leq\left[\iint_{G} \rho^{2}(w) d u d v\right]^{1 / 2}\left[\iint_{D} T_{\nu} d \xi d \eta\right]^{1 / 2}
$$

where

$$
\nu=\operatorname{Belt}[F]
$$

Proof. We use the notation $d F=P d \zeta+Q d \bar{\zeta}$, where $P=\partial F$ and $Q=\bar{\partial} F$.

We can suppose that $T_{\nu}$ is defined and finite a.e. on $D$, because otherwise the right-hand side of $(\mathrm{C} 2)$ is infinite. With the definition of $T_{\nu}$ in mind, this means that $P=Q$ a.e. on $A$, where $A$ is the set on which Jacobian $J_{F}$ equals zero.

Since $F$ is absolutely countinuous on $\Gamma_{x}$ for a.e. $x \in(\alpha, \beta)$, we find

$$
\rho-\operatorname{length}\left(\gamma_{x}\right)=\int_{\Gamma_{x}}(\rho \circ F)(\zeta)|P \| 1-\nu| d \eta
$$

By Fubini's theorem and assumption (C1),

$$
\operatorname{area}(D) \leq \iint_{D}(\rho \circ F)(\zeta)|P \| 1-\nu| d \xi d \eta
$$

Since

$$
J_{F}=|P|^{2}\left(1-|\nu|^{2}\right) \text { a.e. on } D,
$$

the term on the right can be written in the form

$$
\tau=\iint_{D}(\rho \circ F)(\zeta) J_{F}^{1 / 2} T_{\nu}^{1 / 2} d \xi d \eta
$$

Next, using the Cauchy-Schwarz inequality we conclude that

$$
\tau \leq A^{1 / 2} \cdot B^{1 / 2}
$$

where

$$
A=\iint_{D}\left(\rho^{2} \circ F\right)(z) J_{F}(z) d x d y \quad \text { and } \quad B=\left[\iint_{D} T_{\nu} d \xi d \eta\right]
$$

Let

$$
C=\iint_{G} \rho^{2}(w) d u d v
$$

We need the following lemma to finish the proof.

Lemma 2. $A \leq C$. 
Proof. Let the measure $\mu$ be defined by

$$
\mu(E)=\int_{E} J_{F}(z) d x d y
$$

and

$$
\mu_{F}(E)=m(F(E)),
$$

for every Lebesgue measurable set $E$.

Since $F$ is a homeomorphism which possesses finite partial derivatives a.e. in $D$, by Lemma 3.3 ([LV], p. 131)

$$
\mu(E) \leq \mu_{F}(E)
$$

and therefore we have the desired result.

\section{Proof of a new version of the Main Inequality}

There are a number of papers of Reich and Strebel which concern various forms of the Main Inequality. Our proof is based on their ideas.

Here, we give a complete proof of Theorem 1, because we need to be careful when we work with mappings whose dilatation is not bounded. For the reader's convenience, let us recall the statement of Theorem 1 .

Theorem 1. Suppose that

(a) $f$ is a homeomorphism of $\bar{\Delta}$ onto itself,

(b) $f$ has generalized first derivatives on $\Delta$, and

(c) $f$ is the identity on $\partial \Delta$.

Then the inequality

$$
\iint_{\Delta}|\varphi(z)| d x d y \leq \iint_{\Delta}|\varphi(z)| T_{\mu} \varphi(z) d x d y
$$

holds for every integrable analytic function $\varphi$ on $\Delta$.

D1. First, observe that Theorem 1 can be reduced to the case in which $\varphi$ is also analytic on $\partial \Delta$. Indeed, for $0<r<1$ let $\varphi_{r}$ be the function defined by $\varphi_{r}(z)=\varphi(r z), z \in \Delta$. If Theorem 1 holds for every $\varphi_{r}$ then, letting $r$ approach 1 , we conclude that the theorem holds for $\varphi$, by Lebesgue's dominated convergence theorem.

Suppose now that $\varphi$ is an analytic function in $\bar{\Delta}$. The following decomposition is possible (see [S] and [S1]). Up to a set of Lebesgue 2-dimensional measure zero, 
$\Delta=\bigcup_{k=1}^{n} \Sigma_{k}$, where $\left\{\Sigma_{k}\right\}$ are disjoint simple connected "strip" domains. Each $\Sigma_{k}$ is swept out by a family of vertical trajectories of the holomorphic quadratic differential $\varphi(z) d z^{2}$, and in each $\Sigma_{k}$ there exists a single-valued schlicht branch $\zeta=\Phi_{k}(z)$ of $\int \sqrt{\varphi(z)} d z$. Each region $D_{k}=\Phi_{k}\left(\Sigma_{k}\right)$ is vertically convex.

In [S], it is merely assumed that $\varphi$ is analytic on $\Delta$, instead of $\partial \Delta$, so that countably many, instead of merely finitely many $\sum_{k}$ can occur. Actually, in our use of the strip domains, the advantage of limiting ourselves to finitely many is purely didactic. For the local and global behaviour of the trajectories of holomorphic quadratic differentials we refer the reader to Strebel's book ( [S2]).

The following fact is important in the proof of Theorem 1.

D2. The vertical trajectories of a holomorphic quadratic differential are globally geodesics in Teichmüller's metric

$$
d s^{2}=|\varphi(z)||d z|^{2}
$$

Note that $\zeta=\Phi_{k}(z)$ is a single-valued branch of $\int \sqrt{\varphi} d z$ in $\Sigma_{k}$, that $D_{k}=$ $\Phi_{k}\left(\Sigma_{k}\right)$ and $\Gamma_{x}=\Gamma_{x}^{k}$ is the interval which is the intersection of $D_{k}$ with the straight line $\operatorname{Re} z=x$.

Let $\theta_{x}=\Phi_{k}^{-1}\left(\Gamma_{x}\right)$ and $G_{k}=f\left(\Sigma_{k}\right)$. Thus $\theta_{x}$ is the trajectory of the holomorphic quadratic differential $\varphi(z) d z^{2}$. Let $\gamma_{x}=f\left(\theta_{x}\right)$.

Since $\theta_{x}$ is a global geodesic in the Teichmüller metric,

$$
\begin{gathered}
d s^{2}=|\varphi(z)||d z|^{2} \\
\operatorname{length}\left(\Gamma_{x}\right)=\int_{\theta_{x}}|\varphi(z)|^{1 / 2}|d z| \leq \int_{\gamma_{x}}|\varphi(w)|^{1 / 2}|d w| .
\end{gathered}
$$

Thus we can apply Lemma 1 to the functions $F_{k}=f \circ \Phi_{k}^{-1}$ and $\rho(w)=|\varphi(w)|^{1 / 2}$. Hence, by Lemma 1 ,

$$
\iint_{\sum_{k}}|\varphi(z)| d x d y=\operatorname{area}\left(D_{k}\right) \leq A_{k} B_{k},
$$

where

$$
A_{k}=\left[\iint_{G_{k}}|\varphi(w)| d u d v\right]^{1 / 2}
$$

and

$$
B_{k}=\left[\iint_{D_{k}} T_{\nu} d \xi d \eta\right]^{1 / 2}, \quad \nu=\nu_{k}=\operatorname{Belt}\left(F_{k}\right)
$$


Using the change of variables $z=\Phi_{k}^{-1}(\zeta)$, we get

$$
B_{k}=\iint_{\sum_{k}}|\varphi(z)| T_{\mu} \varphi(z) d x d y
$$

Further application of the Cauchy-Schwarz lemma and (D1) give

$$
\sum_{k=1}^{n}\left(\varphi-\operatorname{area}\left(\Sigma_{k}\right)\right) \leq \sum_{k=1}^{n} A_{k} B_{k} \leq A \cdot B
$$

where

$$
A=\left(\sum_{k=1}^{n} A_{k}^{2}\right)^{1 / 2} \text { and } B=\left(\sum_{k=1}^{n} B_{k}^{2}\right)^{1 / 2} .
$$

Now, Theorem 1 follows from the fact that

$$
A=\left[\iint_{\Delta}|\varphi(z)| d x d y\right]^{1 / 2} \text { and } B=\left[\int_{\Delta}|\varphi| T_{\mu} \varphi d x d y\right]^{1 / 2},
$$

where $\mu=\operatorname{Belt}[f]$.

\section{E. The uniqueness results}

E1. The following example may serve to explain the definition of harmonic mappings (see below).

Example 2. Let $0<\epsilon<1$ and let $J$ be an open set in $(0,1)$ of measure $\epsilon$, which is dense in $(0,1)$. Define the function $v$ by

$$
v(y)=\int_{0}^{y} K_{G}(t) d t, \quad y \geq 0
$$

where $G=\bigcup_{n=0}^{\infty}(J+n)$ and $K_{G}$ is the characteristic function of $G$.

Put $f(z)=x+i v(y)$ and $F=[0, \infty) \backslash G$. We leave to the reader to check the following facts:

(a) $f$ is a homeomorphism of the upper half plane $H$ onto itself;

(b) $f$ is a conformal mapping on $R \times G$; and

(c) the set $R \times F$ has infinite Lebesgue 2-dimensional measure, and its image under $f$ has measure zero.

Let $M$ and $N$ be two Riemann surfaces and let $\rho$ be a measurable function on $N$ which satisfies $\rho>0$ a.e. on $N$. A function $f: M \mapsto N$ is called harmonic, 
with respect to $\rho$ (metric density) if the expression $\varphi(z) d z^{2}$, where $\varphi(z)=\tau \circ f p \bar{q}$, is equal a.e. to a holomorphic quadratic differential on $M$, where $\tau=\rho$ a.e. on $N$.

For a similar definition, see $[\operatorname{Re} 1],[\operatorname{Re} 2]$ and $[\operatorname{ReS} 2]$.

Note that we do not require that $\rho$ be integrable over $N$. Also, observe that the definition does not require any smoothness of the metric density $\rho$ on the image surface $N$, while this is required in order to write the Euler-Lagrange equation (see (A2)). From now on, when we say harmonic, we mean harmonic with respect to some metric density $\rho$ in the sense of this last definition.

Let $\tau$ be an arbitrary Borel measurable function on $H$ which is positive a.e. on $H$, let $\varphi$ be the conformal mapping of $\Delta$ onto $H$ and let $\psi$ be the inverse function of $\varphi$. Define the metric density $\rho$ on $\Delta$ by $\rho(w)=\tau(\varphi(w))\left|\varphi^{\prime}(w)\right|$ and let $g=\psi \circ f \circ \varphi$. It is easy to verify that $g$ is a harmonic homeomorphism of $\Delta$ onto itself, with respect to the metric density $\rho$, which is the identity on $\partial \Delta$ but is not the identity on $\Delta$.

This example shows why we need the Hopf differential of $f$ to be nonzero in condition (e) of Theorem 2 (see below).

In order to give motivation for the proofs of Theorem 2 and Theorem 3, the following consideration is useful.

E2. The case when $f=I d$ on $\partial \Delta$. Suppose that $f$ is a harmonic diffeomorphism of $\bar{\Delta}$ onto itself and that the Hopf differential of $f$ is integrable on $\Delta$. Then the Beltrami dilatation $\mu$ of $f$ has the form

$$
\mu(z)=s(z) \frac{\overline{\varphi(z)}}{|\varphi(z)|},
$$

where $s$ is a non-negative measurable function and $\varphi=\rho \circ f p \bar{q}$ is an analytic integrable function on $\Delta$. Thus, we see that the expression $\mu \varphi /|\varphi|$, which appears in the main inequality, is equal to $|\mu|$; and an application of the Main Inequality to the analytic function $\varphi$ gives

$$
\int_{\Delta}|\varphi| d x d y \leq \int_{\Delta}|\varphi| \frac{1-|\mu|}{1+|\mu|} d x d y .
$$

This inequality implies that if $\varphi$ is not identically 0 , that $\mu=0$ a.e. in $\Delta$. Hence we conclude that $f$ is a conformal mapping and, since $f=I d$ on $\partial \Delta$, that $f=I d$ on $\Delta$.

E3. Proof of Theorem 2. For the convenience of the reader, let us recall the statement of Theorem 2 and a few facts concerning harmonic mappings.

Suppose that $f$ has $L^{1}$-derivatives in a domain $D$ and that $f$ is a harmonic mapping with respect to the metric $\rho$ on $D$. Let $\mu$ be the Beltrami dilatation and $\varphi$ the Hopf differential of $f$. Then 
(a) $J_{f}=0 \operatorname{iff}|\varphi| p=\varphi q$,

(b) $\mu=|\mu||\varphi| / \varphi$.

Note that this expression for the Beltrami dilatation $\mu$ is the key for applying the Main Inequality to harmonic mappings.

Theorem 2. Suppose that

(a) $f$ is a homeomorphism of $\bar{\Delta}$ onto itself,

(b) $f$ has generalized first derivatives on $\Delta$,

(c) $f$ is the identity on $\partial \Delta$,

(d) $f$ is harmonic with respect to the metric density $\rho$, and

(e) the Hopf differential of $f$ is nonzero and integrable on $\Delta$.

Then $f$ is the identity on $\bar{\Delta}$.

Remark. If $H o p f(f)=0$ on $\Delta$, Theorem 2 is also true under the additional hypothesis that $f$ maps sets of measure zero onto sets of measure zero.

Proof of Remark. The assumption that $f$ has the generalized first derivatives implies that $f$ has finite partial derivatives a.e. in $\Delta$. Since $f$ is a homeomorphism, it is differentiable a.e. in $\Delta$ by the Gehring-Lehto lemma. Hence, we conclude that $J_{f} \geq 0$ a.e. in $\Delta$, because $f$ is a sense preserving mapping.

Let $A$ be the set on which $q$ is defined and is equal to zero. The assumption $\varphi=0$ on $\Delta$ implies that $p=0$ a.e. on $A^{c}=\Delta \backslash A$. Since $|p| \geq|q|$ a.e. in $\Delta$, we conclude that $q=0$ a.e. in $\Delta$. Next, the assumption that $f$ has generalized first derivatives and Weyl's lemma imply that $f$ is equal to an analytic function a.e. in $\Delta$. Using the boundary condition and continuity of $f$, it is easy to verify that $f$ is the identity on $\Delta$.

Proof of Theorem 2. Let $B$ denote the set on which $J_{f}$ is defined and equals zero. The expression $T_{\mu} \varphi$ which appears in the statement of Theorem 1 must be defined appropriately on the set $B$.

Since $f$ is harmonic, $\mu \varphi /|\varphi|$ equals $|\mu|$ a.e. on $\Delta$. Using this as motivation (see the definition of $T_{\mu} \varphi$ ), we can define $T_{\mu} \varphi$ to be zero on $B$. Hence, $T_{\mu} \varphi$ is defined a.e. on $\Delta$ and

$$
T_{\mu} \varphi=\frac{1-|\mu|}{1+|\mu|}
$$

Now, an application of Theorem 1 to the analytic function $\varphi$ gives

$$
\int_{\Delta}|\varphi| d x d y \leq \int_{\Delta}|\varphi| \frac{1-|\mu|}{1+|\mu|} d x d y .
$$

As in $\mathrm{E} 2$, one can conclude that $\mu=0$ a.e. on $\Delta$, and hence that $f$ is the identity on $\Delta$. 
E4. Corollaries of the Main Inequality. In order to prove Theorem 3, we need a few lemmas.

First, let us recall the hypotheses of Theorem 3:

(a) $f$ and $g$ are homeomorphisms of $\bar{\Delta}$ onto itself, and $f=g$ on $\partial \Delta$;

(b) $f$ and $g$ are locally quasiconformal on $\Delta$;

(c) $f$ and $g$ are harmonic mappings with respect to some metric density $\rho$ on $\Delta$; and

(d) the Hopf differentials of $f$ and $g$ are integrable on $\Delta$.

We use the notation $\mu=\operatorname{Belt}[f], \tilde{\mu}=\operatorname{Belt}\left[f^{-1}\right]$ and $\tilde{\chi}=\operatorname{Belt}\left[g^{-1}\right]$.

Also, for convenience we write $\zeta=f(z), k=k(\zeta)=|\mu(z)|=|\bar{\mu}(\zeta)|$ and $s=s(\zeta)=|\tilde{\chi}(\zeta)|$.

The following result is a corollary of Theorem 1.

Lemma 1. With the notation above and the hypotheses stated for Theorem 3 ,

$$
\int_{\Delta}|\varphi| d x d y \leq \int_{\Delta}|\varphi| \frac{1-|\mu(z)|}{1+|\mu(z)|} \frac{1+s(\zeta)}{1-s(\zeta)} d x d y
$$

where $\varphi=\rho \circ f p \vec{q}$.

Proof. By hypothesis, $g^{-1} \circ f$ is the identity on $\partial \Delta$ and $g^{-1} \circ f$ is locally quasiconformal on $\Delta$ and hence has $L^{2}$-derivatives. An application of the new version of the Main Inequality to $g^{-1} \circ f$ and $\varphi$ gives

$$
\int_{\Delta}|\varphi| \leq \int_{\Delta}|\varphi| T_{\mu} \varphi \frac{|1-\tilde{\chi}(\zeta) \theta \varphi||\varphi|^{2}}{1-|\tilde{\chi}(\zeta)|^{2}} d x d y
$$

where

$$
\theta=\frac{\bar{p}}{p} \overline{(1-\mu \varphi /|\varphi|)}(1-\mu \varphi /|\varphi|)^{-1} \quad \text { and } \quad p=\partial f .
$$

Recall that $\mu \varphi /|\varphi|=|\mu|$, and therefore $\theta=\bar{p} / p$. By the triangle inequality

$$
|1-\tilde{\chi} \theta \varphi /| \varphi|| \leq 1+|\tilde{\chi}(\zeta)|
$$

hence we obtain (E1).

For our purpose, it is convenient to use the change of variables $\zeta=f(z)$ in (E1) and express the inequality (E1) in terms of the metric density $\rho$ instead of $\varphi$. Using this change of variables, we obtain

$$
|\varphi| d x d y=\left|\varphi \circ f^{-1}\right|\left|J_{f-1}(\zeta)\right| d \xi d \eta
$$


Let $\tilde{\varphi}(\zeta)=|\varphi(z)| / J_{f}(z)$. Since $|\varphi(z)|=\rho(\zeta)|p||q|$, we have

$$
\tilde{\varphi}(\zeta)=\rho(\zeta) \frac{k(\zeta)}{1-k^{2}(\zeta)}
$$

This gives the following result.

Lemma 2. With the above notation and the hypotheses stated in Theorem 3 ,

$$
\int_{\Delta} \rho \frac{k}{1-k^{2}} d \xi d \eta \leq \int_{\Delta} \rho \frac{k}{1-k^{2}} \frac{1-k}{1+k} \frac{1+s}{1-s} d \xi d \eta
$$

where $\rho, k$ and $s$ are the functions of $\zeta$ defined at the beginning of item $\mathrm{E} 4$.

Note that Lemma 1 and Lemma 2 are valid without the assumption that $g$ is a harmonic mapping.

E5. Proof of Theorem 3 and Theorem 4. Note that the inequality (E4) holds if $k$ and $s$ change roles. If we sum the corresponding inequalities, we obtain

$$
\int_{\Delta} \alpha \rho \leq \int_{\Delta} \beta \rho
$$

where

$$
\alpha=\frac{k}{1-k^{2}}+\frac{s}{1-s^{2}}, \quad \beta=\frac{k}{(1+k)^{2}} \frac{1+s}{1-s}+\frac{s}{(1+s)^{2}} \frac{1+k}{1-k} .
$$

We need the following lemma to finish the proof.

Lemma 3. $\alpha \geq \beta$.

The proof of this lemma is elementary and follows from the fact that

$$
\alpha-\beta=2(k-s)(f(k)-f(s))[(1-k)(1-s)]^{-1},
$$

where $f(x)=x(1+x)^{-2}$ is an increasing function of $x \in[0,1)$.

Lemma 3 with (E5) actually show that $\alpha=\beta$, i.e., $k=s$.

Thus we have equality in (E1) and therefore equality in (E3). This means that $\tilde{\chi} \theta \varphi /|\varphi|$ is non-positive a.e. in $\Delta$. Since

$$
-\tilde{\mu}(\zeta) \cdot \frac{\bar{p}}{p}=\mu(z)
$$

we conclude that $\tilde{\chi} / \tilde{\mu}$ is positive a.e. in $\Delta$, i.e., $\tilde{\chi}=\tilde{\mu}$ a.e. in $\Delta$. This, finally, gives $f=g$ in $\bar{\Delta}$. 
Since the Reich-Strebel inequality holds on Riemann surfaces of finite analytic type, we can use the same method as in the proof of Theorem 3 to obtain the following result.

Theorem 4. (Second uniqueness property). Let $M$ and $N$ be two compact Riemann surfaces of the same genus $g \geq 2$. Suppose that $f$ and $g$ are harmonic quasiconformal mappings from $M$ onto $N$, which are homotopic to one other. If, in addition, the Hopf differentials of $f$ and $g$ are integrable on $\Delta$, then $f \equiv g$.

Proof. Using the same procedure and notation as in the proof of Theorem 3, we conclude that $\tilde{\mu}=\tilde{\chi}$ a.e. on $R^{\prime}$. Hence, $h=f^{-1} \circ g$ is a conformal mapping of $R$ onto itself, which is homotopic to the identity. If genus $g \geq 2$, then by Theorems $\mathrm{A}$ and $\mathrm{B}$ (see below) $h$ must be the identity!

We would like to call attention to the difference between this proof and that of Theorem 1 at this point.

To finish the proof, we require the following results.

Theorem A. (Theorem 5.2 [L], p. 157). The covering group $G$ of the upper half-plane over a compact Riemann surface is finitely generated and of the first kind.

This means that the limit set $L$ of $G$ is the whole real axis and, in particular, that the covering group $G$ is non-elementary, i.e., the limit set $L$ contains more than two points.

Theorem B. (Theorem 1.3 [L], p. 179). Let $R$ be a Riemann surface with a non-elementary covering group. If $f: R \mapsto R$ is a conformal mapping homotopic to the identity, then $f$ is the identity mapping.

Combining these theorems, we conclude that $h$ is the identity and therefore $f \equiv g$ on $R$.

\section{F. Further results and comments}

We give an application of Theorem 3 in a case in which the energy integral is infinite.

F1. Suppose that

(a) $f$ and $g$ are harmonic diffeomorphisms from the $\Delta$ onto itself with respect to the Poincare metric,

(b) the Hopf differentials $\varphi=\operatorname{Hopf}(f)$ and $\psi=\operatorname{Hopf}(g)$ are integrable on $\Delta$.

Since $\varphi$ and $\psi$ belong to the Bers space (see, for example, [Ah], [W] and [AMM] for the definition and properties of the Bers space), a result of Wan [W] 
shows that $f$ and $g$ are quasiconformal mappings of $\Delta$ onto itself. If, in addition, we suppose that $f=g$ on the boundary of the unit disk, an application of Theorem 3 shows that $f$ and $g$ are identical.

Note that every harmonic diffeomorphism of $\Delta$ onto itself with respect to the Poincaré metric has infinite energy integral.

The following example shows that, without the assumption that the Hopf differentials are integrable, Theorem 2 is not valid.

F2. Let $\varphi$ be a conformal mapping of the unit disk $\Delta$ onto the upper half-plane $H$ and let $\rho(w)=\left|\varphi^{\prime}(w)\right|$. Next, let $g=\psi \circ h \circ \varphi$, where $\psi$ is the inverse function of $\varphi$ and $h$ is given by $h(z)=x+i k y, k>0$. We leave the reader to verify that $g$ is a quasiconformal harmonic mapping (with respect to $\rho$ ) of the unit disk $\Delta$ onto itself and that $g=I d$ on the boundary of $\Delta$.

Although the metric defined by the density $\rho$ is flat on the complex plane $C$ except at one point, Theorem 2 is not valid.

F3. In connection with parts (F1) and (F2) of this section, there is an interesting conjecture which is due to Schoen (see also [Sc]).

Conjecture. The quasiconformal harmonic homeomorphisms from the unit disk $\Delta$ onto itself, with respect to the Poincare metric, are parametrized by the boundary values of quasiconformal maps of the disk.

This is a question which involves proving both an existence and a uniqueness theorem. The existence result for this ideal boundary value problem has been shown by $\mathrm{Li}$ and Tam ([LT1]) under the additional hypothesis that the boundary map is sufficiently differentiable. They have also obtained counterexamples to uniqueness without the quasiconformal hypothesis (but with continuity) and then proved the uniqueness part of Schoen's conjecture (see [LT2]).

A result of Wan ([W]) gives a parametrization of the quasiconformal harmonic homeomorphisms of $\Delta$ in terms of bounded holomorphic quadratic differentials on $\Delta$. Wan has shown that if $f$ is a quasiconformal mapping, then the Hopf differential of $f$ is bounded with respect to the Poincaré metric on $\Delta$. Conversely, for any bounded holomorphic quadratic differential $\Phi$ on $\Delta$, there is a unique quasiconformal harmonic homeomorphism $f: \Delta \mapsto \Delta$ such that $\operatorname{Hopf}(f)=\Phi$.

F4. In this section, we give an example of a harmonic homeomorphism whose Jacobian is zero on a set of positive measure.

Let $\varepsilon>0$ be arbitrary and let $G$ be an open set in $R$ of measure $\varepsilon$ which contains all rational numbers. Let $h$ be the characteristic function of $G$ and let $v$ 
be the function defined by

$$
v(x)=\int_{-\infty}^{x} h(t) d t, \quad x \in R .
$$

Put $f(z)=2 x+i v(y)$ and let $\rho$ be the metric density defined by $\rho(f(z))=4$ for $y \in G$ and $\rho(f(z))=3$ for $y \in K$, where $K$ is the complement of $G$.

We leave the reader to check the following facts.

(a) $f$ is a homeomorphism of $C$ onto $R \times(0, \varepsilon)$, which has $L^{\infty}$-derivatives,

(b) $f$ is a harmonic mapping with respect to metric density $\rho$ on $C$,

(c) the Jacobian $J_{f}$ is equal zero a.e. on $R \times K$.

Finally, we are going to state some results communicated by the second author at the Symposium Contemporary Mathematics (see [M]).

F5. Theorem 3 remains valid if condition (b) (in the hypothesis of Theorem 3, item E3) is replaced by the following.

(e) $f, g$ and their inverse mapping have $L^{2}$-derivatives.

The idea of the proof is as follows. If the condition (e) holas, then one can conclude that $f \circ g^{-1}$ and $g \circ f^{-1}$ have $L^{1}$-derivatives and these partial derivatives satisfy the chain rule (for details see Lemma 6.4 [LV], p. 151). Hence, we can apply Theorem 1 to these functions as in the proof of Lemma 1 of item E4.

It is well-known that condition (b) implies condition (e) (see, for example, [LV]).

Also, Theorem 4 is valid under weaker conditions. Instead of the assumption that $f$ and $g$ are quasiconformal mappings from $M$ onto $N$, it is sufficient to suppose that $f$ and $g$ are homeomorphisms from $M$ onto $N$, which satisfy condition (e).

For a development of the theory of harmonic mappings by means of Sobolev spaces, we refer to [SY].

F6. Harmonic maps and extremal QC mapping. Before we state the results, we require some notation.

Suppose that $f$ is a quasiconformal mapping of the unit disk $\Delta$ onto itself. Let

$$
k[f]=\operatorname{ess} \sup \left\{\left|\mu_{f}(z)\right|: z \in \Delta\right\}
$$

and let $Q(f)$ denote the collection of all quasiconformal mappings of $\Delta$ whose pointwise boundary values on $\partial \Delta$ agree with those of $f$. We call $f$ extremal (in its Teichmüller class) if $k[f] \leq k[g]$ for every $g \in Q(f)$. An extremal quasiconformal mapping $f$ is uniquely extremal (in its Teichmüller class) if $k[f]<$ $k[g]$ for every other $g$ in $Q(f)$. 
Theorem 5. (First removable singularity theorem). Suppose that

(a) $f$ is a quasiconformal mapping from $\Delta$ onto $\Delta$;

(b) $f$ is a harmonic function with respect to the metric density $\rho$ on $\Delta \backslash K$, where $K$ is compact subset of $\Delta$;

(c) $f$ is extremal in its Teichmüller class; and

(d) there are two positive constants $m$ and $M$, such that $m \leq|\varphi(z)| \leq M$ for each $z \in \Delta \backslash K$, where $\varphi$ is the Hopf differential of $f$.

Then $\varphi$ has an analytic extension $\tilde{\varphi}$ from $\Delta \backslash K$ to $\Delta$; and

$$
\mu(z)=k|\tilde{\varphi}(z)| / \tilde{\varphi}(z) \text { a.e. in } \Delta
$$

where $k$ is a constant.

Theorem 6. (Second removable singularity theorem). Suppose that

(a) $f$ is a uniquely extremal quasiconformal mapping in its class, from $\Delta$ onto $\Delta ;$ and

(b) $f$ is a harmonic function with respect to the metric density $\rho$ on $\Delta \backslash K$, where $K$ is compact subset of $\Delta$.

Then we have the same conclusion as in the previous theorem.

During our work with Božin on the problems related to uniquely extremal quasiconformal mappings (see [BMM]), we also obtained some results of this type.

Acknowledgement. The authors would like to thank Professors Kurt Strebel and Edgar Reich for their continuous encouragement and support, and their useful comments concerning this paper.

\section{REFERENCES}

[Ah] L. V. Ahlfors, Lectures on Quasiconformal Mappings, Princeton University Press, 1966.

[AMM] I. Anić, V. Marković and M. Mateljević, Uniformly bounded maximal $\Phi$-disks, Bers spaces and harmonic maps, Proc. Amer. Math. Soc., to appear.

[BLMM] V. Božin, N. Lakić, V. Marković and M. Mateljević, Unique extremality, J. Analyse Math. 75 (1998), 299-338.

[BMM] V. Božin, V. Marković and M. Mateljević, Unique extremality in the tangent space of Teichmüller space, Proceedings of the International Conference "Generalized FunctionsLinear and Nonlinear Problems", Novi Sad, Aug. 31-Sept. 4, 1966; Integral Transforms and Special Functions 6 (1997), 223-227.

[CH] J.M. Coron and F. Helein, Harmonic diffeomorphisms, minimizing harmonic maps and rotational symmetry, Compositio Math. 69 (1989), 175-228.

[G] F. P. Gardiner, Teichmüller Theory and Quadratic Differentials, Wiley-Interscience, New York, 1987.

[J] J. Jost, Two Dimensional Geometric Variational Problems, Wiley, New York, 1991, pp. 1-236. 
[L] O. Lehto, Univalent Functions and Teichmüller Spaces, Springer-Verlag, New York, 1987.

[LT1] P. Li and L. F. Tam, The heat equation and harmonic maps of complete manifolds, Invent. Math. 105 (1991), 1-46.

[LT2] P. Li and L. F. Tam, Uniqueness and regularity of proper harmonic maps II, Indiana Univ. Math. J. 42 (1993), 593-635.

[LV] O. Lehto and K. I. Virtanen, Quasiconformal Mapping, Springer-Verlag, Berlin, New York, 1965.

[M] M. Mateljević, Some geometric questions related to harmonic maps, Symposium Contemporary Mathematics, University of Belgrade, 18-20 Dec. 1988.

[MM1] M. Mateljević and V. Marković, The unique extremal QC mapping and uniqueness of HahnBanach extensions, Mat. Vesnik 48 (1996), 107-112.

[MM2] V. Marković and M. Mateljević, New version of the Grötzsch principle and Reich-Strebel inequality, Mat. Vesnik 49 (1997), 235-239.

[MM3] M. Mateljević and V. Marković, Reich-Strebel inequality, harmonic mappings and uniquely extremal q.c. mappings, Nevanlinna Colloquium, Lausanne 1997 (Abstract).

[Rel] E. Reich, A generalized Dirichlet integral, J. Analyse Math. 30 (1976), 456-463.

[Re2] E. Reich, On the variational principle of Gerstenhaber and Rauch, Ann. Acad. Sci. Fenn. 10 (1985), 469-475.

[Re3] E. Reich, The unique extremality counterexample, J. Analyse Math. 75 (1998), 339-347.

[ReS1] E. Reich and K. Strebel, On quasiconformal mappings which keep the boundary points fuxed, Trans. Amer. Math. Soc. 136 (1969), $211-222$.

[ReS2] E. Reich and K. Strebel, On the Gerstenhaber-Rauch principle, Israel J. Math. 57 (1987), $89-100$.

[Sc] $\mathrm{R}$. Schoen, The role of harmonic mappings in rigidity and deformation problems, Lecture Notes in Pure and Appl. Math. 143 (1993), 179-200.

[SY] R. Schoen and S. T. Yau, Lectures on Harmonic Maps, Conf. Proc. and Lecture Notes in Geometry and Topology, Vol. II, International Press, Cambridge, MA, 1997.

[S] K. Strebel, Zur Frage der Eindeutigkeit extremaler quasikonformer Abbildungen des Einheitskreises II, Comment. Math. Helv. 39 (1964), 77-89.

[S1] K. Strebel, On quasiconformal mappings of open Riemann surfaces Comment. Math. Helv. 53 (1978), 301-321

[S2] K. Strebel, Quadratic Differentials, Springer-Verlag, Berlin, 1984.

[W] T. Wan, Constant mean curvature surfaces, harmonic maps and universal Teichmüller space, J. Differential Geom. 35 (1992), 643-657.

[We] H. B. Wei, On the uniqueness problem of harmonic mappings, Proc. Amer. Math. Soc. 124 (1996), 2337-2341

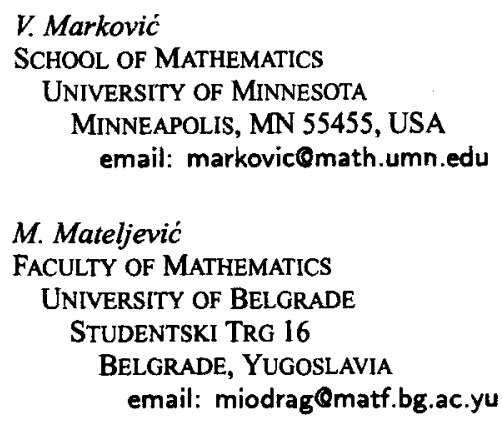

(Received April 12, 1999 and in revised form October 1, 1999) 